\title{
REPRESENTATIONS OF THE DISCRETE HEISENBERG GROUP AND COCYCLES OF AN IRRATIONAL ROTATION
}

\author{
Larry Baggett, Wesley E. Mitchell, and Arlan Ramsay
}

Introduction. One of the most familiar examples in ergodic theory is that of an irrational rotation of a circle. These rotations are well understood in many ways, but continue to be studied in their appearances outside ergodic theory proper. For example, we mention their uses in $C^{*}$-algebras $[4,25,26,30]$, function algebras $[10,12]$ and unitary representations of groups $[15,16,22,23,28]$. The main purpose of this paper is to present some information about (one-) cocycles (defined below) of irrational rotations in the context of representation theory, and to raise a few questions in that same context.

The existence of irreducible cocycles of every dimension is known from general arguments $[28,32,35]$, and explicit formulas have been given in some cases $[1,5,13,15]$. Our interest is in specific formulas, just as specific formulas are sought when studying group representations. Indeed, we derive cocycle formulas from formulas for representations of the discrete Heisenberg group, and then use the cocycles to get formulas for representations of the Mautner group. Thus we are closely tied to representation theory in our motivation and in our method. Besides the explicitness of the cocycle formulas, we want to emphasize that they arise in a systematic way in the representation theory setting.

Cocycles appear in the representation theory of locally compact groups (taken here to be second countable) when forming induced representations. Let $G$ be a locally compact group with a (right) Borel action on a compact metric space $X$. If $\mathcal{U}$ is the unitary group of a Hilbert space $\mathcal{K}$, a Borel function $S: X \times G \rightarrow \mathcal{U}$ such that $S\left(x, g_{1} g_{2}\right)=S\left(x, g_{1}\right) S\left(x g_{1}, g_{2}\right)$ for $x \in X, g_{1}, g_{2} \in G$ is called a cocycle for the action. If $X \times G$ is given its natural groupoid structure, such an $S$ is a homomorphism of $X \times G$ to $U$, i.e. a representation of $X \times G[22,23,27,28]$. Suppose $\mu$ is a finite Borel measure on $X$, quasi-invariant and ergodic for the action of $G$, and $\nu$ is a finite measure equivalent to Haar measure on $G$. By an intertwining between two cocycles $S$ and $S^{\prime}$ for the action of $G$ on $X$, relative to $\mu$, we mean an operator-valued function $A$ on $X$ such that $A(x) S(x, g)=S^{\prime}(x, g) A(x g)$ for $\mu \times \nu$ almost all pairs $(x, g)$ in $X \times G$. Two such cocycles $S$ and $S^{\prime}$ are equivalent if there exists a unitary-valued intertwining between them, and a single cocycle $S$ is called irreducible (relative to $\mu$ ) if the only intertwinings between $S$ and itself are the scalar operator-valued functions.

The groups of interest to us here are semidirect products $G=N K$ where $N$ is normal and abelian. Via inner automorphism, there are actions of both $G$ and $K$

Received June 13, 1983.

Research by the first author and the last author was supported in part by the National Science Foundation.

Michigan Math. J. 31 (1984). 
on the dual $\hat{N}$ of $N$. If $\mu$ is a finite measure on $\hat{N}$, which is ergodic and quasiinvariant for the action of $G$ (equivalently for the action of $K$ ), then there is a one-one correspondence between the set of equivalence classes of irreducible cocycles $S$ for the action of $K$ on $\hat{N}$ relative to $\mu$, and the set of equivalence classes of irreducible unitary representations $U$ of $G$ for which the projectionvalued measure associated by Stone's theorem to $\left.U\right|_{N}$ has the same null sets as $\mu$. See [21] and [28]. This correspondence can be described in two stages as follows:

1. There exists a "special" one-dimensional cocycle $M$, for the action of $G$ on $\hat{N}$ relative to $\mu$, such that the map $S \rightarrow M \otimes(S \cdot \pi)$ ( $\pi$ the natural map of $G$ onto $K$ ) defines a one-one correspondence between the set of equivalence classes of irreducible cocycles $S$ for the action of $K$ relative to $\mu$, and the set of equivalence classes of irreducible cocycles $R$ for the action of $G$ relative to $\mu$.

2. Let $R$ be a cocycle for the action of $G$ on $\hat{N}$ relative to $\mu$, and suppose that $R$ takes values in the unitary group on a Hilbert space $H(R)$. Because $\mu$ is quasiinvariant for the action of $G$, there exists a nonnegative function $\rho$ on $\hat{N} \times G$ such that the following formula defines a unitary representation $U$ of $G$ on the Hilbert space $L^{2}(\mu ; H(R))$ :

$$
\left[U_{g} f\right](x)=\rho(x, g)[R(x, g)](f(x g)) .
$$

This $U$ is called the representation of $G$ induced by $R$, and it is denoted $U=$ $\operatorname{IND}(R)$. The map $R \rightarrow \operatorname{IND}(R)$ defines a one-one correspondence between the set of equivalence classes of irreducible cocycles $R$ for the action of $G$ on $\hat{N}$ relative to $\mu$, and the set of equivalence classes of irreducible unitary representations $U$ of $G$ for which the projection-valued measure associated to $\left.U\right|_{N}$ has the same null sets as $\mu$.

The one-one correspondence $S \rightarrow \operatorname{IND}[M \otimes(S \cdot \pi)]$ is useful in both directions. From information about cocycles we can deduce facts about representations, and vice versa.

In this paper we study two groups. In one of them the group $K$ is $\mathbf{Z}$, the measures $\mu$ are concentrated on circles and we have irrational rotations. In the other $K=\mathbf{R}$, the measures $\mu$ are concentrated on 2-tori and the action is an irrational winding.

In $\S 1$ we define the discrete Heisenberg group and use some of its representations to get formulas for cocycles of an irrational rotation, by means of the oneone correspondence just described. These cocycles belong to a certain class for which we can describe the cohomology relation in terms of the parameters involved, and this is done in $\S 2$. There we show how the formulas of $\S 1$ fit with those of Bagchi, Mathew and Nadkarni [1], with those of Ismagilov [13], with one of I. Brown [5] and some of S. Kawakami [15]. In $\$ 3$ we use an equivalence of an irrational rotation with an irrational flow on a 2-torus to transfer cocycles to the flow. Then we use these to make representations of the Mautner group, this being a standard example of a group for which such flows arise. Finally, $\S 4$ contains some questions related to the material in the earlier sections.

Here is some notation used throughout the paper: $\mathbf{Z}=$ the integers, $\mathbf{R}=$ the real numbers, $\mathbf{C}=$ the complex numbers, $\bar{T}=\{z \in \mathbf{C}:|z|=1\}$ (called "the circle"). 
1. Construction of irreducible cocycles. Let $\mu$ be normalized Lebesgue measure on $\bar{T}$ and choose an element $\rho \in \bar{T}$ of infinite order. (Then $\mathbf{Z}$ can act on $\bar{T}$ by the formula $\alpha \cdot n=\alpha \rho^{n}$, an irrational rotation.) Our version of the discrete Heisenberg group comes from a 2-cocycle $\sigma$ on $\mathbf{Z}^{2}$ defined by $\sigma\left(p_{1}, q_{1} ; p_{2}, q_{2}\right)=$ $\rho^{-p_{1} q_{2}}$. We make a group $G=\left(\mathbf{Z}^{2}\right)^{\sigma}$ from the set $\bar{T} \times \mathbf{Z}^{2}$ in the standard manner: $\left(\alpha_{1}, p_{1}, q_{1}\right)\left(\alpha_{2}, p_{2}, q_{2}\right)=\left(\alpha_{1} \alpha_{2} \sigma\left(p_{1}, q_{1} ; p_{2}, q_{2}\right), p_{1}+p_{2}, q_{1}+q_{2}\right)$. Then part of the dual of $G$ is in one-one correspondence with the $\sigma$-dual of $\mathbf{Z}^{2}$. We are interested in constituents of a certain direct integral decomposition of the $\sigma$-regular representation, regarded as a representation of $G$, described as follows:

Let $d=\left(d_{1}, d_{2}\right)$ be a pair of relatively prime integers, in which we take $d_{1}$ to be positive to simplify the discussion. For each $\lambda \in \bar{T}$ such that $\lambda^{d_{1}}=\rho$ and each pair $(\xi, \eta) \in \bar{T}^{2}$, define a representation $V=V^{\xi, \eta, d}$ of $G$, acting in $L^{2}(T, \mu)$, by

$$
\left(V_{\omega, p, q} f\right)(\alpha)=\omega \xi^{p} \eta^{q} \lambda^{d_{1} p q+(1 / 2) d_{2} q^{2} \alpha^{d_{1} p+d_{2} q}} f\left(\alpha \lambda^{q}\right) .
$$

These are the constituents mentioned above, and we study their restrictions to two particular normal subgroups, namely $N_{(1,0)}=\{(\omega, p, 0): \omega \in \bar{T}, p \in \mathbf{Z}\}$ and $N_{(0,1)}=\{(\omega, 0, q): \omega \in \bar{T}, q \in \mathbf{Z}\}$. Each of these is abelian and has a dual isomorphic to $\mathbf{Z} \times \bar{T}$. The following theorem is a direct consequence of the results of [24].

THEOREM 1.1. Each $V^{\xi, \eta, d}$ is an irreducible representation of $G . V^{\xi, \eta, d}$ is unitarily equivalent to $V^{\xi^{\prime}, \eta^{\prime}, d^{\prime}}$ if and only if $d=d^{\prime}$ and there is a $j \in \mathbf{Z}$ such that $\xi^{d_{2}} \eta^{-d_{1}}=\left(\xi^{\prime}\right)^{d_{2}}\left(\eta^{\prime}\right)^{-d_{1}} \rho^{j}$. Further, the retriction of $V^{\xi, \eta, d}$ to $N_{(1,0)}$ (respectively $N_{(0,1)}$ ) has a projection valued measure on the dual, $\mathbf{Z} \times \bar{T}$, which has uniform multiplicity $d_{1}$ (respectively $d_{2}$ ) and whose null sets are those of the product of a point mass at 1 on $\mathbf{Z}$ with $\mu$ on $\bar{T}$.

REMARK. Of course, $\lambda$ is a parameter which we are holding fixed in this discussion. We shall see in $\S 2$ that $\lambda$ can be absorbed into the parameter $\eta$.

Given $\left(\xi_{0}, \eta_{0}, d\right)$, choose $\theta \in \bar{T}$ so that $\theta=\xi_{0}^{d_{1}}$, and define $A: L^{2}(\bar{T}) \rightarrow L^{2}(\bar{T})$ by $(A f)(\alpha)=f(\alpha \bar{\theta})$. Then $A$ is a unitary equivalence of $V^{\xi_{0}, \eta_{0}, d}$ onto $V^{1, \eta, d}$, where $\eta=\theta^{-d_{2}} \eta_{0}$. We will work with these representations, and delete the superscript 1 .

Our first goal is to write $V=V^{\eta, d}$ as an induced representation $U^{R}$, where $R$ is a representation of the virtual group $\bar{T} \times G$, and where $(\omega, p, q)$ in $G$ acts on $\alpha$ in $\bar{T}$ by $\alpha \cdot(\omega, p, q)=\alpha \rho^{q}$.

By the Corollary of Lemma 3 of [24], $V \mid N_{(1,0)}$ is equivalent to a direct sum of $d_{1}$ copies of $\chi_{1} L$, where $\chi_{1}$ is the identity character of $\bar{T}$ and $L$ is the regular representation of $\mathbf{Z}$. Thus we want to find a representation equivalent to $V$ acting in the Hilbert space $\mathcal{H C}=L^{2}\left(\bar{T}, \mathbf{C}^{d_{1}}\right)$, which is a space on which a representation of $G$ induced by a cocycle of $\bar{T} \times G$ is naturally defined.

Now $V$ is defined on $L^{2}(\bar{T})$, and we can define a unitary operator $C: L^{2}(\bar{T}) \rightarrow \mathfrak{H C}$ as follows: If $f \in L^{2}(\bar{T})$ then $f$ has a Fourier expansion $f(\alpha)=\sum_{k=-\infty}^{\infty} c_{k} \alpha^{k}$. Let $C f: \bar{T} \rightarrow \mathbf{C}^{d_{1}}$ be defined by

$$
(C f)_{l}(\alpha)=\sum_{k=-\infty}^{\infty} c_{d_{1} k+l} \alpha^{k}
$$


for $1 \leqslant l \leqslant d_{1}$. For $f \in \mathcal{H}$, with components $f_{1}, \ldots, f_{d_{1}},\left(C^{-1} f\right)(\alpha)=\sum_{j=1}^{d_{1}} \alpha^{j} f_{j}\left(\alpha^{d_{1}}\right)$. Hence, for $(\omega, p, q) \in G$,

$$
\begin{aligned}
\left(V_{\omega, p, q} C^{-1} f\right)(\alpha) & =\omega \eta^{q} \lambda^{d_{1} p q+(1 / 2) d_{2} q^{2}} \alpha^{d_{1} p+d_{2} q} \sum_{j=1}^{d_{1}} \alpha^{j} \lambda^{q j} f_{j}\left(\left(\alpha \lambda^{q}\right)^{d_{1}}\right) \\
& =\omega \eta^{q} \lambda^{-(1 / 2) d_{2} q^{2}} \sum_{j=1}^{d_{1}}\left(\alpha \lambda^{q}\right)^{j+d_{1} p+d_{2} q} f_{j}\left(\left(\alpha \lambda^{q}\right)^{d_{1}}\right) .
\end{aligned}
$$

We need to find a $g \in \mathcal{F}$, with components $g_{l}$, so that this iunction of $\alpha$ is $\left(C^{-1} g\right)(\alpha)=\sum_{l=1}^{d_{1}} \alpha^{l} g_{l}\left(\alpha^{d_{1}}\right)$. The terms $\alpha^{d_{1} p}$ and $f_{j}\left(\left(\alpha \lambda^{q}\right)^{d_{1}}\right)$ are functions of $\alpha^{d_{1}}$, so we need to understand the terms $\alpha^{j+d_{2} q}$.

For each $q$, choose $m_{q}(j)$ in $\mathbf{Z}$ and $l_{q}(j)$ in $\left\{1, \ldots, d_{1}\right\}$ so that $j+d_{2} q=$ $d_{1} m_{q}(j)+l_{q}(j)$. Then $l_{q}(j+1)=l_{q}(j)+1$ unless $l_{q}(j)=d_{1}$, when $l_{q}(j+1)=1$. Also $m_{q}(j+1)=m_{q}(j)$ unless $l_{q}(j)=d_{1}$, when $m_{q}(j+1)=m_{q}(j)+1$. Let $P_{q}$ be the permutation matrix with $(i, j)$ entry equal to $\delta_{i, l_{q}(j)}$. Let $D_{q}(\alpha)$ be the (diagonal) matrix with $(i, j)$ entry equal to $\delta_{i j} \lambda^{q j} \alpha^{m_{q}(j)}$. Then we have

$$
\begin{aligned}
\left(V_{(\omega, p, q)}^{\eta, d} C^{-1} f\right)(\alpha) & =\omega \eta^{q} \lambda^{-(1 / 2) d_{2} q^{2}} \sum_{j=1}^{d_{1}}\left(\alpha \lambda^{q}\right)^{d_{1}\left(p+m_{q}(j)\right)+l_{q}(j)} f_{j}\left(\left(\alpha \lambda^{q}\right)^{d_{1}}\right) \\
& =\omega \eta^{q} \lambda^{-(1 / 2) d_{2} q^{2}} \sum_{j=1}^{d_{1}} \alpha^{l} g_{l}\left(\alpha^{d_{1}}\right),
\end{aligned}
$$

where

$$
\begin{aligned}
g_{l}(\alpha) & =\sum_{j=1}^{d_{1}} \delta_{l, l_{q}(j)} \lambda^{q l}\left(\alpha \lambda^{d_{1} q}\right)^{p+m_{q}(j)} f_{j}\left(\alpha \lambda^{d_{1} q}\right) \\
& =\sum_{j=1}^{d_{1}} \delta_{l, l_{q}(j)} \lambda^{q\left(d_{1} p+j+d_{2} q\right)} \alpha^{p+m_{q}(j)} f_{j}\left(\alpha \lambda^{d_{1} q}\right) .
\end{aligned}
$$

Thus $C V_{(\omega, p, q)}^{\eta, d} C^{-1} f=g$, where $g$ has components $g_{l}$, and in matrix terms we have

$$
g(\alpha)=\omega \eta^{q} \lambda^{d_{1} p q+(1 / 2) d_{2} q^{2}} \alpha^{p} P_{q} D_{q}(\alpha) f\left(\alpha \lambda^{d_{1} q}\right) .
$$

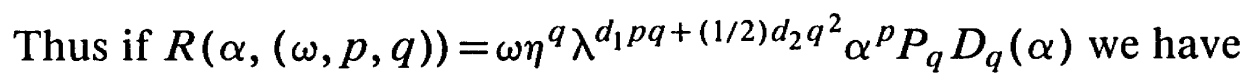

$$
\left(C V_{(\omega, p, q)}^{\eta, d} C^{-1} f\right)(\alpha)=R(\alpha,(\omega, p, q)) f\left(\alpha \lambda^{d_{1} q}\right) .
$$

A calculation shows that

$$
R\left(\alpha,\left(\omega_{1}, p_{1}, q_{1}\right)\right) R\left(\alpha \cdot\left(\omega_{1}, p_{1}, q_{1}\right),\left(\omega_{2}, p_{2}, q_{2}\right)\right)=R\left(\alpha,\left(\omega_{1}, p_{1}, q_{1}\right)\left(\omega_{2}, p_{2}, q_{2}\right)\right) .
$$

THEOREM 1.2. Each $R$ is an irreducible $d_{1}$-dimensional cocycle of the action of $G$ on $\bar{T}$, relative to Lebesgue measure $\mu$. Two such cocycles $R$ and $R^{\prime}$ are equivalent if and only if $d=d^{\prime}$ and $\eta^{d_{1}}=\left(\eta^{\prime}\right)^{d_{1}} p^{j}$ for some $j \in \mathbf{Z}$.

Proof. The calculations preceding the definition of $R$ show that $V^{\eta, d}$ is unitarily equivalent to the induced representation $U^{R}$. The proof then follows from Theorem 9.1 of [28] and Theorem 1.1.

We would like to break up each of these cocycles $R$ as a tensor product of cocycles on $T \times G$ and $T \times \mathbf{Z}$. Define 


$$
\begin{gathered}
M(\alpha,(\omega, \rho, q))=\omega \alpha^{p} \lambda^{d_{1} p q} \\
S(\alpha, q)=\eta^{q} \lambda^{(1 / 2) d_{2} q^{2}} A^{\prime}(\alpha, q)
\end{gathered}
$$

where $A^{\prime}(\alpha, q)=P_{q} D_{q}(\alpha)$.

THEOREM 1.3. $M$ is a one-dimensional cocycle of $\bar{T} \times G . S$ is an irreducible $d_{1}$-dimensional cocycle of $\bar{T} \times \mathbf{Z}$, and $R=M \otimes S$. Two such cocycles $S$ and $S^{\prime}$ are equivalent if and only if $d_{1}=d_{1}^{\prime}, d_{2}=d_{2}^{\prime}$, and $\eta^{d_{1}}=\left(\eta^{\prime}\right)^{d_{1}} \rho^{j}$ for some $j \in \mathbf{Z}$.

Proof. These statements follow from elementary calculations, Theorem 1.2, and Theorem 9.3 of [28].

Thus, for every positive integer $d_{1}$, we have exhibited an uncountable family of inequivalent irreducible $d_{1}$-dimensional cocycles of $T \times \mathbf{Z}$. Indeed, the fact that rotation by $\rho$ is ergodic for Lebesgue measure implies that we have a set of equivalence classes of representations which is not even countably separated, and this is done for every finite dimension $d_{1}$.

2. Other cocycle constructions. In this section we mention some other ways of obtaining specific formulas for cocycles and compare these lists to the one in Section $\S 1$, in the sense of equivalence. These formulas are due to Ismagilov, to Bagchi, Mathew and Nadkarni, to I. Brown, and to S. Kawakami. B. Brencken has found formulas of the same type using $C^{*}$-algebras.

First we recall that a cocycle $U$ for an integer action can be constructed simply by giving the function $U(\bullet, 1)$. The difficulty is in analyzing the equivalences among cocycles, or rather in giving formulas for which this analysis can be readily carried out. Ismagilov [13] proposes the following scheme for any space $X$ and transformation $T$ : Let $P$ be the matrix of a cyclic permutation of the basis in $\mathbf{C}^{n}$. Let $D$ be the diagonal matrix with entries $d_{1}(x), \ldots, d_{n}(x)$ and let $U(x, 1)=P D(x)$. Then $U(x, k)=P D(x) P D(T x) \cdots P D\left(T^{k-1} x\right)$ for $x \in X$ and $k \geqslant 1$, and a similar formula for $k<0$ follows from $U(x,-k)^{-1}=U(x \bullet(-k), k)$. Since the $d_{i}$ are arbitrary, no generality is lost by taking $P$ to correspond to the $n$-cycle $(123 \cdots n)$. Then $U(x, n)$ is diagonal with entries $v_{1}, \ldots, v_{n}$, where

$$
v_{j}(x)=d_{j-1}(x) d_{j-2}(T x) \cdots d_{1}\left(T^{j-2} x\right) d_{n}\left(T^{j-1} x\right) \cdots d_{j}\left(T^{n-1} x\right),
$$

denoting the transformation taking $x$ to $x \cdot 1$ by $T$. If $A: X \rightarrow \mathcal{L}\left(\mathbf{C}^{n}\right)$ commutes with $U$, i.e. $A(x) U(x, k)=U(x, k) A(x \cdot k)$ for $x \in X$ and $k \in \mathbf{Z}[27,28]$, then taking $k=n$ gives $A_{i j}(x) v_{j}(x)=v_{i}(x) A_{i j}(x \bullet n)$. Thus if $T^{n}$ is ergodic the entries $A_{i i}$ must be constant functions. If $i \neq j$ implies that $v_{i}$ and $v_{j}$ are not equivalent under $T^{n}$, then $A_{i j}=0$ for $i \neq j$. Taking $k=1$ in the equation above we see that $A_{i+1, j+1}$ is a multiple of a shift of $A_{i j}$ (taking subscripts modulo $n$ ). Thus, once we know $A_{11}$ is a constant and $A_{1 i}=0$ for $i=2, \ldots, n$, we know $A$ is a constant scalar operator. Thus $U$ is irreducible, provided $T^{n}$ is ergodic and that $i \neq 1$ implies $v_{i}$ and $v_{1}$ are not equivalent relative to $T^{n}$. The converse is clear.

For an irrational rotation, Ismagilov suggests taking the $d_{i}$ 's to be eigenfunctions. Suppose then that $c_{1}, \ldots, c_{n} \in \bar{T}$ and $p_{1}, \ldots, p_{n} \in \mathbf{Z}$ and set $c_{0}=c_{1} c_{2} \cdots c_{n}$, $p_{0}=p_{1}+\cdots+p_{n}$. If $d_{j}(\alpha)=c_{j} \alpha^{p_{j}}$ and $T \alpha=\alpha \rho$ for some $\rho \in \bar{T}$, then the diagonal entry of $U(\alpha, n)$ is $v_{j}(\alpha)=c_{o} \rho^{q_{j}} \alpha^{p_{0}}$, where 


$$
q_{j}=p_{j-2}+2 p_{j-3}+\cdots+(j-2) p_{1}+(j-1) p_{n}+\cdots+(n-1) p_{j} .
$$

There is a non-zero function $f$ satisfying $a \alpha^{p_{0}} f(\alpha)=b \alpha^{p_{0}} f\left(T^{n} \alpha\right)$ (a.e.) if and only if $a / b$ is an eigenvalue for $T^{n}$. Since we are using Lebesgue measure on $\bar{T}$, $T^{n}$ is ergodic and its point spectrum consists of the powers of $\rho^{n}$. Thus we see that the cocycle in question is irreducible if and only if none of the integers $q_{2}-q_{1}, \ldots, q_{n}-q_{1}$ is a multiple of $n$. Each $q_{j}$ is a $\mathbf{Z}$-linear combination of $p_{1}, \ldots, p_{n}$, with coefficients a cyclic permutation of $0,1, \ldots, n-1$. Hence $q_{j}-q_{1}$ is always a $\mathbf{Z}$-linear combination of $p_{1}, \ldots, p_{n}$ with coefficients whose sum is 0 . In $q_{1}$ the coefficient of $p_{1}$ is $n-1$ and in the other $q_{j}$ 's the coefficients $n-2, \ldots, 1,0$ all occur. Hence the coefficients of $p_{1}$ in $q_{2}-q_{1}, \ldots, q_{n}-q_{1}$ are $-1,-2, \ldots,-(n-1)$ in some order. In particular, if $p_{2}=\cdots=p_{n}=0$, then one of $q_{2}-q_{1}, \ldots, q_{n}-q_{1}$ is a multiple of $n$ if and only if $p_{1}$ and $n$ have a non-trivial common factor. In this case $p_{0}=p_{1}$, and we shall see that this cocycle $U$ is equivalent to one with $p_{2}=\cdots=p_{n}=0$, so that $U$ is irreducible if and only if $\operatorname{gcd}\left(p_{0}, n\right)=1$.

To show this, take $A(\alpha)$ to be a diagonal matrix with entry $\alpha^{k_{i}}$ in the $i$ th place. Then a cocycle $U_{1}$ equivalent to $U$ is determined by $U_{1}(\alpha, 1)=$ $A(\alpha) P D(\alpha) A(\alpha \cdot 1)^{-1}=P\left(P^{-1} A(\alpha) P\right) D(\alpha) A(\alpha \cdot 1)^{-1}=P D_{1}(\alpha)$, where $D_{1}(\alpha)$ is diagonal with $i$ th diagonal entry equal to $\rho^{-k_{i}} c_{i} \alpha^{p_{i}+k_{i+1}-k_{i}}$. Thus $D_{1}(\alpha)$ has new powers of $\alpha$, which can be chosen freely subject to the condition that the sum of the changes, which is $k_{2}-k_{1}+k_{3}-k_{2}+\cdots+k_{1}-k_{n}$, be 0 . In particular we can change $p_{1}$ to $p_{0}$ and all the others to 0 . Now $U$ is irreducible if and only if $U_{1}$ is, and $U_{1}$ is irreducible if and only if $n$ does not divide any of $p_{0}, 2 p_{0}, \ldots,(n-1) p_{0}$, and this happens if and only if $\operatorname{gcd}\left(p_{0}, n\right)=1$.

To complete the study of equivalence among the cocycles on Ismagilov's list, we first compare two cocycles in which only the first power of $\alpha$ is allowed to be different from 0 . Thus we have constants $c_{1}, \ldots, c_{n} \in \bar{T}$ and $p_{1} \in \mathbf{Z}$ for $D$, and $c_{1}^{1}, \ldots, c_{n}^{1} \in \bar{T}$ and $p_{1}^{1} \in \mathbf{Z}$ for $D_{1}$, and form $U, U_{1}$ by $U(\alpha, 1)=P D(\alpha), U_{1}(\alpha, 1)=$ $P D_{1}(\alpha)$. Let $c_{0}=c_{1} c_{2} \cdots c_{n}, c_{0}^{1}=c_{1}^{1} c_{2}^{1} \cdots c_{n}^{1}$, and take $q_{j}, q_{j}^{1}$ as before. Now $U(\alpha, n)$ and $U_{1}(\alpha, n)$ are diagonal, and we let $v_{i}$ and $v_{i}^{1}$ be these functions of $\alpha$. If $U$ and $U_{1}$ are equivalent, there is by definition a matrix-valued Borel function $A$ such that $A(\alpha) U(\alpha, 1)=U_{1}(\alpha, 1) A(\alpha \cdot 1)$ for almost all $\alpha$. Hence $A(\alpha) U(\alpha, n)=U_{1}(\alpha, u) A(\alpha \bullet n)$ for almost all $\alpha$, and for all $i, j$ we have

$$
A_{i j}(\alpha) c_{0} \rho^{q_{j}} \alpha^{p_{1}}=c_{0}^{1} \rho^{q_{i}^{1}} \alpha_{1}^{p_{1}^{1}} A_{i j}\left(\alpha \rho^{n}\right) .
$$

Now suppose $f$ is a bounded Borel function and there exists $b \in T$ and $k \in \mathbf{Z}$ such that $f\left(\alpha \rho^{n}\right)=b \alpha^{k} f(\alpha)$ for all $\alpha$. By comparing coefficients in Fourier series we find that $k=0, b$ is a power of $\rho^{k}$, and $f(\alpha)$ is a power of $\alpha$. Hence $p_{1}^{1}=p_{1}$ if $A \neq 0$. Thus $q_{j}=q_{j}^{1}$, which implies that the integers $0, q_{2}-q_{1}^{1}, \ldots, q_{n}-q_{1}^{1}$ are in distinct congruence classes modulo $n$. Hence at most one of the functions $A_{11}, A_{12}, \ldots, A_{1 n}$ can be non-zero. Of course this is already evident from the fact that the two cocycles are irreducible. However, it is also true that as long as $c_{0} / c_{0}^{1}$ is a power of $\rho$ there will be one $i$ for which $\left(c_{0} / c_{0}^{1}\right) \rho^{q_{i}-q_{1}}$ is a power of $\rho^{k}$, in which case there will exist a nonzero $A_{1 i}$. Hence $U$ and $U_{1}$ are equivalent if and only if $p_{1}=p_{1}^{1}$ and $c_{0} / c_{0}^{1}$ is a power of $\rho$. Notice that if we start with two arbitrary 
$D$ and $D_{1}$ and shift so that $p_{2}=\cdots=p_{n}=p_{2}^{1}=\cdots=p_{n}^{1}=0$ we change $c_{0}$ and $c_{0}^{1}$ only by multiplying by a power of $\rho$, and we do not change $p_{0}$ and $p_{0}^{1}$. Thus in general we can say $U \equiv U_{1}$ if and only if $p_{0}=p_{0}^{1}$ and $c_{0} / c_{0}^{1}$ is a power of $\rho$.

In $\S 1$ we derived cocycles $S$ depending on integers $d_{1}$ and $d_{2}$ (relatively prime), a parameter $\eta \in \bar{T}$, and a $d_{1}$-st root of $\rho, \lambda$. Since $d_{2}$ is relatively prime to $d_{1}$, the permutation matrix which occurs there corresponds to an $n$-cycle and hence is conjugate to the one corresponding to $(1,2, \cdots n)$. Thus each $S$ from $\S 1$ has the form described by Ismagilov. Conversely, $\eta$ can be chosen to arrange any value for $c_{0}$ within the class of $S$ 's. Furthermore, we will shortly show that the integer $d_{2}$ is the $p_{0}$ of our analysis above, and since $d_{2}$ can be any integer relatively prime to $d_{1}\left(d_{1}=n\right)$, any cocycle of the form suggested by Ismagilov is equivalent to an $S$ from $\S 1$. To find the value of $p_{0}$ for $S$, notice that the powers of $\alpha$ which occur are $m_{1}(1), m_{1}(2), \ldots, m_{1}\left(d_{1}\right)$, where $m_{1}$ and $l_{1}$ are functions such that each $l_{1}(j) \in\left\{1,2, \ldots, d_{1}\right\}$ and $j+d_{2}=d_{1} m_{1}(j)+l_{1}(j)$. By the properties of these functions as described before Theorem 1.2, if $d_{2}=d_{1} k+r\left(0 \leqslant r<d_{1}\right)$ then $m_{1}(j)=k$ for $j=1,2, \ldots, d_{1}-r$ and $m_{1}(j)=k+1$ for $j=d_{1}-r+1, \ldots, d_{1}$. Thus $m_{1}(1)+\cdots+m_{1}(d)=k\left(d_{1}-r\right)+(k+1) r=d_{2}$, as claimed.

Bagchi, Mathew and Radkarni calculate one particular cocycle from duality and analytic function theory [1, p. 303]. What they obtain fits into the pattern of Ismagilov by taking each $c_{i}=1$ and $p_{1}=\cdots=p_{n-1}=0, p_{n}=1$. Others may be computable by their methods.

I. Brown defines a cocycle by taking $S\left(e^{i \theta}, 1\right)=e^{i \theta / 2}$ for $0 \leqslant \theta<2 \pi$, and shows by group theoretic methods that it is not equivalent to any given by $U(\alpha, 1)=\alpha^{n}$ [5, p. 24]. Thus for the one-dimensional case there are more cocycles than we derived from the discrete Heisenberg group. This class has been extended even further by $S$. Kawakami [15].

3. Applications to other groups. Using the result quoted in the introduction regarding group extensions, the cocycles found in $\$ 1$ can be used to obtain explicit formulas for representations of groups besides the discrete Heisenberg group. There is a general fact, of which we will give an explicit example, which gives existence results in other cases, namely:

PROPOSITION 3.1. Let $H$ be a separable locally compact group and let $H$ act on a standard Borel space $S$. Suppose there is a $\sigma$-finite measure $\nu$ which is invariant and ergodic under $H$ and for which the action is amenable. Then there is a groupoid homomorphism $\psi$ of $S \times H$ into $\bar{T} \times \mathbf{Z}$ such that $R \circ \psi$ is irreducible relative to $\nu$, whenever $R$ is a cocycle of $\bar{T} \times \mathbf{Z}$ which is irreducible relative to Lebesgue measure on $\bar{T}$.

Proof. This follows from results in $[6,7,8,27,28,29]$.

Combining these ideas, we see that whenever the action of $G / N$ on $\hat{N}$ has a $\sigma$-finite invariant measure, we can theoretically make use of irreducible cocycles of $\bar{T} \times \mathbf{Z}$ to produce irreducible representations of $G$. As an example, let us carry out this process explicitly for the Mautner group. 
Recall that the Mautner group $G$ is the semidirect product $\mathbf{C}^{2} \mathbf{R}$ of two dimensional complex space $\mathbf{C}^{2}$ with the real line $\mathbf{R}$. Multiplication in $G$ is given by $(z, w, t)\left(z^{\prime}, w^{\prime}, t^{\prime}\right)=\left(z+z^{\prime} e^{-i t}, w+w^{\prime} e^{-2 \pi i t}, t+t^{\prime}\right)$. With $\mathbf{C}^{2}$ taken as the normal subgroup $N$, we see that $G / N$, which is isomorphic to $\mathbf{R}$, acts on $\hat{N}$ with invariant tori, so we have a virtual group $\bar{T}^{2} \times \mathbf{R}$ where the real number $t$ acts on the pair $(\alpha, \beta)$ in $\bar{T}^{2}$ by $(\alpha, \beta) \cdot t=\left(\alpha e^{i t}, \beta e^{2 \pi i t}\right)$.

A mapping $\psi: \bar{T}^{2} \times \mathbf{R} \rightarrow \bar{T} \times \mathbf{Z}$ as in Proposition 3.1 can be constructed as follows. Parameterize $\beta$ as $e^{2 \pi i \theta}$ for $0 \leqslant \theta<1$. Put $\psi\left(\alpha, e^{2 \pi i \theta}, t\right)=\left(\alpha e^{-i \theta},[\theta+t]\right)$ where $[y]$ indicates the greatest integer in $y$. One checks directly that $\psi$ is a groupoid homomorphism, where $n \in \mathbf{Z}$ acts on $\alpha \in \bar{T}$ by $\alpha \cdot n=\alpha e^{i n}$. In fact, $\psi$ is a reduction homomorphism and thus the conclusion of Proposition 3.1 holds for $\psi$ [27, Theorem $6.17 ; 28$, p. 47;29, Theorem 6.6 and Theorem 7.17]. This can also be verified directly in this case.

Now suppose $\eta \in \bar{T}, d_{1}$ is a pair of relatively prime integers with $d_{1}>0$, and let $\lambda$ be a $d_{1}$-st root of $e^{i}$. Taking notation from $\S 1$, we can define a cocycle $S^{\prime}=$ $S^{\prime(\eta, \lambda, d)}$ of $\bar{T}^{2} \times \mathbf{R}$ of dimension $d_{1}$ by giving the matrix entries

$$
\begin{aligned}
S^{\prime}\left(\alpha, e^{2 \pi i \theta}, t\right)_{k, j} & =S \circ \psi\left(\alpha, e^{2 \pi i \theta}, t\right)_{k, j}=S\left(\alpha e^{-i \theta},[\theta+t]\right)_{k, j} \\
& =\eta^{[\theta+t]} \lambda^{\left([\theta+t]^{2} d_{2} / 2\right)} \delta_{k, l_{[\theta+t]}^{(j)}} \lambda^{j[\theta+t]}\left(e^{-i \theta} \alpha\right)^{m_{[\theta+t]}^{(j)}} .
\end{aligned}
$$

THEOREM 3.2. (i) The cocycle $S^{\prime(\eta, \lambda, d)}$ is irreducible.

(ii) The irreducible cocycles $S^{\prime(\eta, \lambda, d)}$ and $S^{\prime\left(\eta^{\prime}, \lambda^{\prime}, d^{\prime}\right)}$ are equivalent if and only if $d=d^{\prime}$ and $\eta^{d_{1}} \lambda^{(1 / 2) d_{1}\left(d_{1}+d_{2}+1\right)}\left(\left(\eta^{\prime}\right)^{d_{1}}\left(\lambda^{\prime}\right)^{(1 / 2) d_{1}\left(d_{1}+d_{2}+1\right)}\right)^{-1}$ is of the form $e^{\text {in }}$ for an integer $n$.

Proof. This follows from $\S 1$ and the properties of $\psi$.

It remains to describe the irreducible unitary representations of the Mautner Group $\mathrm{G}$ corresponding to these cocycles, according to the method outlined in the introduction.

The tori in $\mathbf{C}^{2}$ are parametrized by pairs of positive real numbers, $r=\left(r_{1}, r_{2}\right)$, and we can write $r \cdot \bar{T}^{2}$ for the torus. If $\eta \in \bar{T}, d=\left(d_{1}, d_{2}\right)$ is a pair of relatively prime integers with $d_{1}$ positive, and $\lambda^{d_{1}}=e^{i}$, define a representation $V$ of $G$ on $L^{2}\left(\bar{T}^{2}, \mathbf{C}^{d_{1}}\right)$ depending on $r, \eta, \lambda$ and $d$ by

$$
\begin{aligned}
\left(\left(V_{z, w, t} f\right)\left(\alpha, e^{2 \pi i \theta}\right)\right)_{k}= & e^{i\left(z, r_{1} \alpha\right)} e^{i\left(w, r_{2} e^{2 \pi i \theta}\right)} \sum_{j=1}^{d_{1}} S^{\prime}\left(\alpha, e^{2 \pi i \theta}, t\right)_{k j} f\left(\alpha e^{i t}, e^{2 \pi i(\theta+t)}\right)_{j} \\
= & e^{i\left(z, r_{1} \alpha\right)} e^{i\left(w, r_{2} e^{\jmath \pi i \theta}\right)} \eta^{[\theta+t]} \lambda^{(1 / 2)[\theta+t]^{2} d_{2}} \\
& \times \lambda^{j[\theta+t]} \sum_{j=1}^{d_{1}} \delta_{k, l_{[\theta+t]}(j)}\left(e^{-i \theta} \alpha\right)^{m_{[\theta+t]}(j)} f\left(\alpha e^{i t}, e^{2 \pi i(\theta+t)}\right)_{j} .
\end{aligned}
$$

THEOREM 3.3. (i) $V$ is irreducible.

(ii) $V$ and $V^{\prime}$ (with primed parameters) are equivalent if and only if $r=r^{\prime}$, $d=d^{\prime}$ and $\left(\eta^{d_{1}} \lambda^{(1 / 2) d_{1}\left(d_{1}+d_{2}+1\right)}\right)\left(\left(\eta^{\prime}\right)^{d_{1}}\left(\lambda^{\prime}\right)^{(1 / 2) d_{1}\left(d_{1}+d_{2}+1\right)}\right)^{-1}$ is of the form $e^{i n}$ for some integer $n$. 
(iii) $V$ restricts on $N,=\mathbf{C}^{2}$, to be a representation which is supported on $r \bar{T}^{2}$, with respect to Lebesgue measure, and which has multiplicity $d_{1}$.

Proof. Parts (i) and (ii) follow from Theorem 3.2 together with [28]. Part (iii) is clear from the formula for the representation.

REMARK. When $d_{1}=1$ these representations $V$ coincide, after a simple reparameterization, with the "new" representations of the Mautner group given in [3]. However, for $d_{1}>1$, the above formulas are the only ones known to us which give representations of the Mautner group satisfying (iii).

\section{Some related questions and remarks.}

A. It would be desirable to have a parametrized family of cocycles of $\bar{T} \times \mathbf{Z}$ which meets every equivalence class of cocycles at least once and for which the equivalence between members of the family can be described in terms of the parameters. Even the part of the space of equivalence classes given by the family in Sections 1 and 2 is not smooth, but something of that sort for the entire space would be useful. Perhaps even this cannot be done. In particular, we mention the lack of explicit formulas for infinite dimensional irreducible cocycles, even though they are known to exist.

B. According to a result of Dye [7,8], many measured groupoids are isomorphic to $\bar{T} \times \mathbf{Z}$ with Lebesgue measure on $\bar{T}$. One example is obtained as follows. Let $\mathbf{Z}_{2}=\mathbf{Z} / 2 \mathbf{Z}$, let $X$ be a countable product of copies of $\mathbf{Z}_{2}$, let $G$ be the subgroup of $X$ consisting of the sequences with only finitely many non-zero components, and let $G$ act on $X$ as a subgroup. Golodets has given an extensive analysis of the cocycles for this groupoid [11] so an explicit isomorphism between it and $\bar{T} \times \mathbf{Z}$ would be useful, as would other explicit isomorphisms.

C. It may be possible to compute explicitly some (or all) of the cocycles given by inner functions according to the method of [1]. Some of these are infinite dimensional, so part of our question in (A) would be answered.

D. There are several papers which establish the existence of measures ergodic for rotation by $\tau$ but singular relative to Lebesgue measure and atom-free $[9,14$, 17, 31]. The formulas in Sections 1 and 2 still define cocycles relative to these measures, and it is natural to ask when the cocycles are irreducible for such a measure $\mu$. The analysis in $\$ 2$ makes it clear that for a cocycle of dimension $n$ the measure $\mu$ must be ergodic for $\tau^{n}$ in order for irreducibility to hold. As explained to us by B. Weiss, the methods of [14] can be used to construct $\mu$ 's which are ergodic for every $\tau^{n}$, and also to construct $\mu$ 's which are ergodic for $\tau$ but not ergodic for some particular $\tau^{k}$. The question of irreducibility for dimension $n$ is equivalent to questions about $L^{\infty}$-spectrum, i.e. the point spectrum of the operator $T$ where $T f(\alpha)=f\left(\alpha \tau^{n}\right)$ for $f \in L^{\infty}(\mu)$. Results of Yoccoz [34] show that this point spectrum is generically uncountable, and a result of Weiss [33] shows that any particular countable set can be forced into the point spectrum by an appropriate choice of $\mu$. Thus it appears that the irreducibility question is non-trivial. 


\section{REFERENCES}

1. S. C. Bagchi, J. Mathew and M. G. Nadkarni, On systems of imprimitivity on locally compact abelian groups with dense actions, Acta Math. 133 (1974), 287-304.

2. L. W. Baggett, Multiplier extensions other than the Mackey extension, Proc. Amer. Math. Soc. 56 (1976), 351-356.

3. - Representations of the Mautner group I, Pacific J. Math. 77 (1978), 7-22.

4. B. Brencken, Representations and automorphisms of the irrational rotation algebra, preprint.

5. Ian D. Brown, Representation of finitely generated nilpotent groups, Pacific J. Math. 45 (1973), 13-26.

6. A. Connes, J. Feldman and B. Weiss, An amenable equivalence relation is generated by a single transformation, Ergodic Theory Dynamical Systems 1 (1981), 431-450.

7. H. Dye, On groups of measure preserving transformations I, Amer. J. Math. 81 (1959), 119-159.

8. — On groups of measure preserving transformations II, Amer. J. Math. 85 (1963), 551-576.

9. E. Effros, Transformation groups and $C^{*}$-algebras, Ann. of Math. (2) 81 (1965), 38-55.

10. T. W. Gamelin, Uniform Algebras, Prentice Hall, Englewood Cliffs, N.J., 1969.

11. V. Ya. Godolets, Classification of representations of the anticommutation relations, Russian Math. Surveys 24 (1969), 1-63.

12. H. Helson, Analyticity on compact abelian groups. Algebras in Analysis (Birmingham, 1973), 1-62, Academic Press, London, 1975.

13. R. S. Ismagilov, On irreducible cycles, related to a dynamic system, Functional Anal. Appl. 3 (1969), 249-251.

14. Y. Katznelson and B. Weiss, The construction of quasi-invariant measures, Israel J. Math. 12 (1972), 1-4.

15. S. Kawakami, Irreducible representations of non-regular semi-direct product groups, Math. Japon. 26 (1981), 667-693.

16. - Representations of the discrete Heisenberg group, Math. Japon. 27 (1982), 551-564.

17. M. Keane, Sur les measures quasi-ergodiques des translations irrationelles, C.R. Acad. Sci. Paris Sér. A-B 272 (1971), A54-A55.

18. W. Krieger, On non-singular transformations of a measure space, I, Z. Wahrsch. Verw. Gebiete 11 (1969), 83-97.

19. - On non-singular transformations of a measure space, II, Z. Wahrsch. Verw. Gebiete 11 (1969), 98-119.

20. _ On quasi-invariant measures in uniquely ergodic systems, Invent. Math. 14 (1971), 184-196.

21. G. W. Mackey, Unitary representations of group extensions, I, Acta Math. 99 (1958), 265-311.

22. - Infinite dimensional group representations, Bull. Amer. Math. Soc. 69 (1963), 628-686.

23. - Ergodic theory and virtual groups, Math. Ann. 166 (1966), 187-207.

24. W. Mitchell, The $\sigma$-regular representation of $\mathbf{Z} \times \mathbf{Z}$, Michigan Math. J. 31 (1984), 259-262.

25. M. Pimsner and D. Voiculescu, Imbedding the irrational rotation $C^{*}$-algebra into an AF algebra, J. Operator Theory 4 (1980), 201-210. 
26. S. Popa and M. A. Rieffel, The Ext groups of the $C^{*}$-algebras associated with irrational rotations, J. Operator Theory 3 (1980), 271-274.

27. A. Ramsay, Virtual groups and group actions, Adv. in Math. 6 (1971), 253-322.

28. - Nontransitive quasiorbits in Mackey's analysis of group extensions, Acta Math. 137 (1976), 17-48.

29. — Subobjects of virtual groups, Pacific J. Math. 87 (1980), 389-454.

30. M. A. Rieffel, $C^{*}$-algebras associated with irrational rotations, Pacific J. Math. 93 (1981), 415-429.

31. K. Schmidt, Infinite invariant measures on the circle. Symposia Mathematica, XXI (Rome, 1975), 37-43, Academic Press, London, 1977.

32. J. J. Westman, Virtual group homomorphisms with dense range, Illinois J. Math. 20 (1976), 41-47.

33. B. Weiss, Measurable dynamics, Proceedings of the S. Kukatani Conference, to appear.

34. J.-C. Yoccoz, Centralisateur d'un difféomorphisme du cercle dont le nombre de rotation est irrationnel, C.R. Acad. Sc. Paris Sér. A-B 291 (1980), A523-A526.

35. R. J. Zimmer, Amenable ergodic group actions and an application to Poisson Boundaries of random walks, J. Funct. Anal. 27 (1978), 350-372.

Wesley E. Mitchell

Department of Mathematics

S.U.N.Y., College at Potsdam

Potsdam, New York 13676

and

Larry Baggett

Arlan Ramsay

Department of Mathematics

University of Colorado

Boulder, Colorado 80309 
